Anton Lavrinienko ${ }^{1 *}$, Jenni Kesäniemi ${ }^{1}$, Phillip C. Watts ${ }^{1}$, Svitlana Serga $^{2}$, Marta Pascual $^{3}$, Francesc Mestres ${ }^{3}$, Iryna Kozeretska $^{2}$

${ }^{1}$ University of Oulu, Department of Ecology, 90014, Oulu, Finland

${ }^{2}$ Taras Shevchenko National University of Kyiv, ESC ñInstitute of Biologyò, Department of General and Molecular Genetics, 01601 Kyiv, Ukraine.

${ }^{3}$ Universitat de Barcelona, Departament de Genètica, Microbiologia i Estadística and IrBio, 08028 Barcelona, Spain.

*Corresponding author: Anton Lavrinienko

Email: Anton.Lavrinienko@oulu.fi (Tel. +358 44 9251411)

Acknowledgments

The authors are grateful to Ivo Tosevski who kindly provide additional information regarding $D$. suzukii diversity and current distribution in Serbia. We also grateful to Valery A. Korneyev and Jaana Jurvansuu, for their helpful suggestions during manuscript preparation. Authors want to thanks the staff of the Department of Biology at Mechnikov National University of Odesa, and the staff of the National Institute of Viticulture and Winemaking, National Academy of Agrarian Sciences of Ukraine, and Alexandra Procenko and Mykhailo Lavrinienko for their invaluable help in material collection. Marta Pascual and Francesc Mestres are part of the research group SGR2014-336 and funded by project CHALLENGEN (CTM2013-48163) of the Spanish Government.

Author Contribution Statement: AL, JK and PW designed the experiments, AL, IK and SS performed the samplings. AL, IK, SS, MP and FM identified the specimens. AL and JK analyzed data and wrote the manuscript. PW supported and assisted the study. All authors read and approved the manuscript.

Abstract

Drosophila suzukii is an invasive polyphagous fruit pest, which has recently emerged as a global threat to agriculture in the Americas and in Europe. Due to the rapid spread, great economic losses and its pest behavior, D. suzukii represents a powerful model for invasion biology and pest management studies. However, despite the current research efforts in Europe, the invasion routes and current distribution, as well as level of genetic diversity in natural populations of D. suzukii still remains unknown. Here, we present the first report of the occurrence of the D. suzukii in Ukraine. Moreover, the sequence of a fragment of the cytochrome oxidase I gene was compared with genetic data on this species collected from populations in Europe, USA and Asia available in public databases. Our data points to an early stage of invasion of $D$. suzukii in Ukraine and reveals a comparatively high genetic diversity in the Ukrainian population of this pest species suggesting a complex invasion scenario from multiple sources. Monitoring the level of genetic variation across space and time as well as understanding the invasion routes of this major invasive insect pest is essential for developing successful pest management strategies.

Key words: cherry fruit fly; invasive species; Spotted Wing Drosophila; population genetics

Key Message

Drosophila suzukii is an invasive agricultural pest that is expanding rapidly worldwide since the late 2000s.

Our data represent the genetic pattern at the start of an invasion by $D$. suzukii in Ukraine.

COI sequence data reveals high genetic diversity in the Ukrainian D. suzukii population.

Our study indicates the potential for multiple sources of D. suzukii invasion into Europe, along with possible recurrent introductions of this major invasive insect pest. 
Invasive species represent significant threats to biodiversity, with impacts on non-native habitats potentially having deleterious ecosystem effects (Crooks 2002; Gurevitch and Padilla 2004) and strong economic burden (Pimentel et al. 2005). Drosophila suzukii (Matsumura, 1931), also known as the spotted wing drosophila (SWD), is an invasive, destructive crop pest, which represents a good example of one of the most severe ongoing biological invasions of the Western Hemisphere (Asplen et al. 2015).

Most drosophilids attack overripe, rotten or damaged fruits, and generally are not considered as pests. Differently, D. suzukii is one of the very few drosophilids that can oviposit in healthy, ripe fruits thus can cause considerable damage to crops (Mitsui et al. 2006; Lee et al. 2011) resulting one of the most damaging pests of cultivated soft-skinned tree fruits and berry crops in the temperate regions (Saguez et al. 2013). Economic losses due to D. suzukii can be enormous; for example, within the USA the estimated annual economic losses attributed to crop damage by $D$. suzukii are in the order of $\$ 700$ million (Bolda et al. 2010; Walsh et al. 2011; Wiman et al. 2016). The fast-paced global spread of D. suzukii, accompanied with a severe economic burden, highlights the importance of tracing current distribution and invasion routes of this novel invasive insect pest.

SWD is native to Asia (Kanzawa 1939; Calabria et al. 2012) but, by 2008, D. suzukii invasion was simultaneously reported in both in the USA (California) (Hauser et al. 2009) and in western Europe (Spain) (Calabria et al. 2012). Since 2008, D. suzukii has expanded its non-native range quite rapidly becoming a key pest species throughout the Americas (Hauser 2011; Deprá et al. 2014; Wang et al. 2016) and in Europe (Cini et al. 2012; Asplen et al. 2015; Arnó et al. 2016). Despite the invasions has been reported at the same time, genetic data indicate that the invasion to North America and Europe by D. suzukii were independent demographic events (Adrion et al. 2014). Interestingly, levels of genetic diversity in the North American populations of $D$. suzukii are quite high, but are relatively low in Europe (and also the island of Hawaii) (Adrion et al. 2014). Low genetic diversity of D. suzukii in Europe compared with populations in the US is hypothesized to be a consequence of a strong genetic bottleneck, yet the idea is based on data from only one European population from Spain (Adrion et al. 2014). More recent genotype data, based on a large $(n=28)$ panel of microsatellite loci, showed similar levels of polymorphism and strong genetic differentiation between a population from France and a population from Hawaii, consistent with the pattern described above (Fraimout et al. 2015). Thus, despite the wealth of genomic data (Chiu et al. 2013; Ramasamy et al. 2016) and present research efforts in many other fields of D. suzukii biology (Hamby et al. 2016), it is still inconclusive whether European D. suzukii populations are characterized by low genetic diversity. Understanding the level of genetic variation in natural populations of $D$. suzukii has a particular importance from the perspective of developing successful global pest management strategies (Haye et al. 2016), as genetic diversity often positively correlates with adaptation (Reed and Frankham 2003; Barrett and Schluter 2008). Moreover, genetic diversity should inform the size and number of independent and/or recurrent introductions that could be occurring, which would inform appropriate management actions.

Although, D. suzukii is the only species of the ómelanogasterô group in Europe that is characterized by (1) a large, pointed and serrated ovipositor in the females, and (2) by a dark spot near the wing tips of adult males, these characters do not allow unambiguous morphological identification during biodiversity surveys by non-specialists. For example, the conspicuous male wing spots require up to 2 days to fully develop, and large serrated ovipositor is similar to that of females of closely-related species such as Drosophila subpulchrella (Hauser 2011). Finally, the immature stages cannot be identified by their morphology. Thus, there is a need to support surveys of Drosophila species with genetic data to provide unambiguous confirmation of the identity of any suspected D. suzukii (Murphy et al. 2016). One of the most widely used methods of identifying of $D$. suzukii is through DNA barcoding: sequence analysis of a fragment of the mitochondrial cytochrome oxidase I (COI) gene (Hebert et al. 2003; Hauser 2011).

Here, we report (1) the Drosophilidae species composition of seven localities distributed across Ukraine, with individuals collected during two years of sampling, (2) the first record of D. suzukii from Ukraine, but only in the most southern locality that was sampled (Yalta, Crimea) and (3) COI gene sequence data that, by comparison with sequenced data available from public databases, indicate high genetic diversity of $D$. suzukii in Ukraine that points to a complex invasion scenario, potentially from multiple sources. Our study sets the baseline of presence/absence data of this species for invasion of Ukraine, which may contribute to further monitoring studies of this pest species and to understand its dispersal behavior. Our study highlights the importance of performing biodiversity surveys and tracking genetic diversity in natural populations of this major invasive insect pest.

Materials and Methods 
Specimens of D. suzukii were found during routine biodiversity surveys for Drosophila species that were undertaken in the summer-autumn seasons of 2014 and 2015. The seven sampling sites throughout Ukraine were: Yalta, Odesa, Umanô Kharkiv, Piryatin, Kyiv and Chornobyl (Fig. 1). According to KLي̣penô slassification most of Ukraine is characterized by a humid continental climate, except for the South of Crimea (i.e. the Yalta sample location), which is subtropical (Köppen W 1931; Kottek et al. 2006).

Fig. 1 Geographical distribution of D. suzukii sampling localities. (a) World map with sampling localities of COI analyzed specimens derived from GenBank (Online Resource HGB1), denoted with a circles. A star symbol represents location with specimens sequenced in the present study. Color of the symbols correspond to the sampling locations in the haplotype network (Fig.2). (b) Seven locations across Ukraine surveyed for Drosophila species. Sampling locations are denoted by open and closed circles, closed circle (Yalta, Crimea) indicates the location where specimens of $D$. suzukii were collected

In each locality, flies were netted over four traps that had been baited with 6-7 smashed fermented apples and about 300 $\mathrm{ml}$ of commercially available wheat beer (Serga et al. 2015). The baited traps made of thick colorful, flexible plastic film, were kept at each location for 72 hours. A brief description of the sampled localities and their GPS coordinates can be found in Serga et al. (2015), with the only difference being the sample site coordinates in Yalta, where sampling during 2014-2015 was performed in Yalta city center in a private garden that had mixed vegetation, fig tree

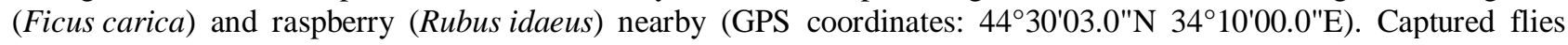
were identified on the basis of their external morphology under a 7x-45x stereomicroscope (Konus Crystal, Italy). Morphological identification of D. suzukii was carried out according to Hauser (2011) and putative specimens preserved at $-20^{\circ} \mathrm{C}$ in $96 \%$ ethanol for molecular analyses.

\section{Molecular methods}

Genomic DNA was extracted from nine individuals of $D$. suzukii (sampled during summer 2015) using a standard high salt method, modified from Aljanabi and Martinez (1997). A 693 bp fragment of the COI mtDNA gene was amplified using the universal primers LCO1490 and HCO2198 (Folmer et al. 1994). Polymerase chain reactions (PCR) were performed in $10 \mu \mathrm{L}$ reactions containing $2 \mu \mathrm{L}$ of DNA, $5 \mu \mathrm{L}$ of $2 \mathrm{X}$ DreamTaq Green PCR Master Mix (Thermo Scientific) and $0.5 \mu \mathrm{M}$ of both forward and reverse primers. Amplification conditions were: $95^{\circ} \mathrm{C}$ for $3 \mathrm{~min}$, then 35 cycles of: $95^{\circ} \mathrm{C}$ for $30 \mathrm{~s}, 55^{\circ} \mathrm{C}$ for $30 \mathrm{~s}$, and $72^{\circ} \mathrm{C}$ for $1 \mathrm{~min}$, followed by $72^{\circ} \mathrm{C}$ for $7 \mathrm{~min}$. For sequencing, the PCR products were purified with Exonuclease I and FastAP Thermosensitive Alkaline Phosphatase (Thermo Scientific), cycle sequenced in both directions using BigDye v.3.1 chemistry (Applied Biosystems), and visualized with an ABI3730 (Applied Biosystems). Sequences were edited manually with BioEdit v.7.2.5 (Hall 1999) and aligned with MEGA6 (Tamura et al. 2013) and then translated to confirm the absence of stop codons. Representative sequences of D. suzukii were deposited in GenBank (KX268719-KX268727).

To infer the genetic relationships of these Ukrainian D. suzukii with other native (e.g. Asia) and invasive (e.g. Europe and the USA) populations of $D$. suzukii, $C O I$ gene sequences for $D$. suzukii were downloaded from GenBank. We only used those COI sequences that (1) corresponded to the same fragment that we sequenced and (2) whose GenBank record contained detailed information about the sampling location. We obtained 74 sequences that represented $D$. suzukii samples from Europe (Portugal, Spain, Italy and Serbia), USA (California, Oregon, Washington and Arkansas), China and Japan (for further details see Online Resource HGB1). A haplotype network was constructed with TCS v.1.21 (Clement et al. 2000) and edited using tcsBU (TCS Beautifier) (Múrias Dos Santos et al. 2016).

Results

Drosophila individuals were collected from seven regions throughout Ukraine, between 2014 and 2015, and identified as belonging to 14 different Drosophila species (see Online Resource SD2). The Drosophilidae species fauna of Ukraine is a quite typical for the Palearctic region (Bächli and Rocha-Pite 1982). D. suzukii was collected during August on both sampling years from Yalta (Crimea) (Table 1), which represents the first record of D. suzukii in Ukraine.

Table 1 Sample locations with GPS coordinates, sampling dates, total number of individuals collected (in parentheses) from the Ukraine during 2014 and 2015

Genomic DNA extraction was obtained from nine wild individuals from Yalta collected in 2015. After sequencing using COI universal primers, we identify 4 different haplotypes. Our study of the genetic diversity of D. suzukii individuals from Ukraine was based on a haplotype network that incorporated an additional 74 D. suzukii COI sequences available from GenBank (see accession numbers in Online Resource HGB1), with 606 bp of the COI gene used in the final alignment (Fig. 2). The haplotype network uncovered substantial genetic diversity, most of which was present in samples from $D$. suzukiiôs putative native range in Japan. Higher amount of COI haplotypes was found in the samples from Ukraine compared to other European countries, similar in level of diversity to the samples from the USA. 
The most frequent haplotype in Europe was also found in individuals from Ukraine as well as in samples from Japan. The second notable feature of our data is that Ukrainian D. suzukii COI haplotypes clustered with both invaded by D. suzukii areas, the samples from Europe and with the samples from the USA (both areas invaded by D. suzukii). In addition, it is worth mentioning that there are one haplotype shared between the USA and Chinese D. suzukii individuals only (Fig. 2). One private haplotype was found in samples from Italy. Most interestingly, the other Ukrainian haplotypes were found in samples from the USA, China and Japan. Similarly, one haplotype from Serbia was found also in samples from the USA and China (Fig. 2), but not including other European locations.

Fig. 2 Haplotype network of COI gene fragment (606 bp) for D. suzukii sampled from Ukraine, Europe, the USA, China and Japan (see GenBank accession numbers and number of samples in Online Resource HGB1). Colors indicate sample locations. Small white circles represent undetected intermediate haplotypes and each line corresponds to a mutational step. The area of the circles represent the amount of identical COI gene sequences used in the alignment

\section{Discussion}

Drosophila suzukii is native to the mountainous temperate regions of East Asia (Kanzawa 1934; Kanzawa 1939; Asplen et al. 2015) and has likely dispersed to Western countries via trade of fruit by sea (Hauser 2011; Calabria et al. 2012). Invasion by $D$. suzukii presents a major agricultural problem, and identifying the routes of invasion, particularly the areas that represent its source, is an important issue from the pest management perspective. Here, we report the invasion of D. suzukii in Ukraine (Yalta, Crimea), with the population having high levels of genetic diversity in contrast to other populations of D. suzukii in Europe.

There are apparently two separate invasion routes into Europe and into the USA (Adrion et al. 2014), however the source (s) of D. suzukii for both regions remains unknown. In the USA, the invasion of D. suzukii could have occurred by a large number of founding individuals and/or from multiple sources, as could be inferred from high genetic diversity and 1 haplotype sharing with both China and Japan. Unfortunately, only 4 COI sequences from $D$. suzukii from China could be included in our analysis, as most of the D. suzukii COI sequence data from China had an ambiguous origin. In contrast, European $D$. suzukii was, up to now, mostly represented by one predominant haplotype, with just another haplotype unique to Italy; this pattern is consistent with a substantial bottleneck during the foundation of European D. suzukii populations (Adrion et al. 2014). Interestingly, the new individuals collected from Yalta (Ukraine) revealed no genetic signature of a sharp bottleneck and shared COI haplotypes with populations in both the USA and Europe, suggesting multiple sources of invasion into the Ukraine by D. suzukii. Alternatively, it is possible that $D$. suzukii had been established in Yalta for a significant period of time and was introduced one or few times with many individuals to maintain a genetically diverse pool. While it is hard to determine the route and timing of the invasion by $D$. suzukii into Crimea, the latter hypothesis seems highly unlikely since all our sampling localities had been surveyed for Drosophila species annually since 2005 (Kozeretska et al. 2008; Radionov et al. 2011; Serga et al. 2014; Serga et al. 2015). The relevance of this survey effort points to the arrival of $D$. suzukii in southern Ukraine close to the summer of 2014. Thus, it seems more likely that D. suzukii was recently introduced to Crimea on multiple occasions from distinct sources, such as from the two introduced areas (i.e. the USA and Europe) or alternatively, from the two ancestral areas (i.e. China and Japan).

A combination of geographical profiling analyses and data on the trade of fresh fruit made by Cini et al. (2014) suggests that the most likely arrival location of D. suzukii in Europe would be the region surrounding Marseilles, southern France, from where it would have subsequently spread across Europe. This idea is supported by the first records of D. suzukii in California and in Spain, both occurring close to important sea ports (Rota-Stabelli et al. 2013; Cini et al. 2014), as well as examples of dispersal of the Drosophila species that follow commercial fruit trafficking routes (Capy and Gibert 2004; Lachaise and Silvain 2004). Indeed, our report of D. suzukii from Yalta, touristic center which has a port and is located on the coast of the Black Sea, is concordant with the idea that trade of fruit by sea routes and travelling industry, presents an important dispersal method by D. suzukii. Interestingly, that port in Yalta annually host hundreds of passenger cruise liners and cargo trade ships from all over the world. Unfortunately, lack of molecular analysis data for specimens found on multiple occasions in other European countries (Asplen et al. 2015), as well as often poor description of already submitted to GenBank files might decrease resolution of D. suzukiiôs population genetics studies. Which proves how important is to report the information correctly, since with wide sampling where the information of the sampling locations is properly stated the future studies can be more complete and precise.

The invasion of D. suzukii in Ukraine we report in the presents great concern, taking into account the enormous economic losses that this species has caused in USA (Wiman et al. 2016), and to some extent in Europe (Asplen et al. 2015). Recent invasions of D. suzukii in some of Eastern European countries (e.g. Hungary, Czech Republic, Slovakia, Bulgaria and Serbia) have shown a rapid population expansion after invasion by apparently quite few individuals (Asplen et al. 2015). For example, since D. suzukii was first reported in Serbia in 2014, it rapidly dominated the drosophilid assemblages across the country (Tosevski et al. 2014). All individuals of D. suzukii sampled across Serbia shared the same haplotype (Tosevski et al. 2014), which was identical to the COI haplotypes identified from Spain, Portugal, Italy and Japan. However, an additional individual collected from southern Serbia in November of 2014 (that 
was sequenced after the first report of D. suzukii from Serbia, GenBank accession number: KX273434) (Tosevski et al. 2014) shares its COI haplotype with D. suzukii from the USA and Chinese populations. This again indicates the potential for multiple sources of $D$. suzukii invasion into Europe, along with potential recurrent introductions of this highly invasive pest species. Thus, in contrast with other European D. suzukii populations as previously argued (Adrion et al. 2014), D. suzukii in Ukraine and Serbia contain comparatively high levels of genetic diversity that potentially offers a new source of genetic variation in Europe. Establishment of an invasive species often benefits from standing genetic variation that allows rapid adaptation to new selection pressures (Frankham 2005; Markert et al. 2010; Bouzat 2010). Potentially, high genetic diversity in D. suzukii populations in Europe can facilitate this species range expansion and increase fruit yield losses caused by this invasive insect pest, possible also even lessening the impact of biological control agents and pest management activities.

Drosophila suzukii populations dynamics mostly depends on temperature, humidity (Tochen et al. 2014, 2016b; Hamby et al. 2016), and the availability of essential food resources (Tochen et al. 2016a). The dry Mediterranean climate, which is similar to that in Yalta, seems not to be preferred by D. suzukii (Hauser et al. 2009; Calabria et al. 2012). However, the apparent absence of $D$. suzukii from continental part of Ukraine may be temporary as these areas have more suitable conditions (i.e. temperate climate, milder summer temperatures and higher humidity), which significantly increase fecundity and longevity in D. suzukii populations (Tochen et al. 2016b). Therefore, the invasion of this pest species into more temperate regions of Ukraine and possible recurrent introductions to other parts of Europe pose a potential threat to agriculture, especially berry crop producers. Currently, it is hard to determine, whether the high level of genetic diversity in D. suzukii populations in Yalta is unique, or whether it is a common pattern across Europe. Further research applying wide range of multilocus genetic markers and more samples from native or invaded D. suzukii areas is needed to provide more resolution. Future studies can make use of the mitochondrial genes, as well as microsatellites markers, which have been shown to be a powerful tool to understand invasion routes and genetic diversity in Drosophila natural populations (Pascual et al. 2007; Bahder et al. 2015). Results presented herein contribute to identification of $D$. suzukii global invasion routes and might help to establish this species as a good model object for research on invasion biology and pest management.

Compliance with Ethical Standards:

Conflict of Interest: The authors declare that they have no conflict of interest.

Ethical approval: All applicable international, national, and institutional guidelines for the care and use of animals in research were followed.

\section{References}

Adrion JR, Kousathanas A, Pascual M, et al (2014) Drosophila suzukii: The genetic footprint of a recent, worldwide invasion. Mol Biol Evol 31:3148ї 3163. doi: 10.1093/molbev/msu246

Aljanabi SM, Martinez I (1997) Universal and rapid salt-extraction of high quality genomic DNA for PCR-based techniques. Nucleic Acids Res 25:4692ї 4693. doi: 10.1093/nar/25.22.4692

Arnó J, Solà M, Riudavets J, Gabarra R (2016) Population dynamics, non-crop hosts, and fruit susceptibility of Drosophila suzukii in Northeast Spain. J Pest Sci 89:713ï 723. doi: 10.1007/s10340-016-0774-3

Asplen MK, Anfora G, Biondi A, et al (2015) Invasion biology of spotted wing Drosophila (Drosophila suzukii): a global perspective and future priorities. J Pest Sci 88:469ï 494. doi: 10.1007/s10340-015-0681-z

Bahder BW, Bahder LD, Hamby KA, et al (2015) Microsatellite Variation of two Pacific Coast Drosophila suzukii (Diptera: Drosophilidae) Populations. Environ Entomol 44:1449ï 53. doi: 10.1093/ee/nvv117

Barrett RDH, Schluter D (2008) Adaptation from standing genetic variation. Trends Ecol. Evol. 23:38ї 44.

Bolda MP, Goodhue RE, Zalom FG (2010) Spotted Wing Drosophila: Potential Economic Impact of a Newly Established Pest. Agric Resour Econ Updat Univ California Giannini Found 13:5ï 8.

Bouzat JL (2010) Conservation genetics of population bottlenecks: the role of chance, selection, and history. Conserv Genet 11:463ï 478. doi: 10.1007/s10592-010-0049-0

Bächli G, Rocha-Pite MT (1982) Annotated bibliography of Palearctic species of Drosophilidae (Diptera). Beitr ge zur Entomol 32:303ї 392.

Calabria G, Máca J, Bächli G, et al (2012) First records of the potential pest species Drosophila suzukii (Diptera: Drosophilidae) in Europe. J Appl Entomol 136:139ï 147. doi: 10.1111/j.1439-0418.2010.01583.x 
Capy P, Gibert P (2004) Drosophila melanogaster, Drosophila simulans: So similar yet so different. In: Genetica. pp 5ї 16

Chiu JC, Jiang X, Zhao L, et al (2013) Genome of Drosophila suzukii, the spotted wing drosophila. G3 3:2257ï 71. doi: $10.1534 / \mathrm{g} 3.113 .008185$

Cini A, Anfora G, Escudero-Colomar LA, et al (2014) Tracking the invasion of the alien fruit pest Drosophila suzukii in Europe. J Pest Sci 87:559ï 566. doi: 10.1007/s10340-014-0617-z

Cini A, Ioriatti C, Anfora G (2012) A review of the invasion of Drosophila suzukii in Europe and a draft research agenda for integrated pest management. Bull Insectology 65:149ї 160.

Clement M, Posada D, Crandall KA (2000) TCS: a computer program to estimate gene genealogies. Mol Ecol 9:1657ї 1659. doi: 10.1046/j.1365-294x.2000.01020.x

Crooks JA (2002) Characterizing ecosystem-level consequences of biological invasions: the role of ecosystem engineers. Oikos 97:153ї 166. doi: 10.1034/j.1600-0706.2002.970201.x

Deprá M, Poppe JL, Schmitz HJ, et al (2014) The first records of the invasive pest Drosophila suzukii in the South American continent. J Pest Sci 87:379ї 383. doi: 10.1007/s10340-014-0591-5

Folmer O, Black M, Hoeh W, et al (1994) DNA primers for amplification of mitochondrial cytochrome c oxidase subunit I from diverse metazoan invertebrates. Mol Mar Biol Biotechnol 3:294ï 299.

Fraimout A, Loiseau A, Price DK, et al (2015) New set of microsatellite markers for the spotted-wing Drosophila suzukii (Diptera: Drosophilidae): A promising molecular tool for inferring the invasion history of this major insect pest. Eur J Entomol 112:855ï 859. doi: 10.14411/eje.2015.079

Frankham R (2005) Genetics and extinction. Biol. Conserv. 126:131ï 140.

Gurevitch J, Padilla DK (2004) Are invasive species a major cause of extinctions? Trends Ecol Evol 19:470ї 474. doi: 10.1016/j.tree.2004.07.005

Hall T (1999) BioEdit: a user-friendly biological sequence alignment editor and analysis program for Windows 95/98/NT. Nucleic Acids Symp. Ser. 41:95ï 98.

Hamby KA, E. Bellamy D, Chiu JC, et al (2016) Biotic and abiotic factors impacting development, behavior, phenology, and reproductive biology of Drosophila suzukii. J Pest Sci 89:605ï 619. doi: 10.1007/s10340-0160756-5

Hauser M, Gaimari S, Damus M (2009) Drosophila suzukii new to North America.

Hauser M (2011) A historic account of the invasion of Drosophila suzukii (Matsumura) (Diptera: Drosophilidae) in the continental United States, with remarks on their identification. Pest Manag Sci 67:1352ï 1357. doi: $10.1002 /$ ps. 2265

Haye T, Girod P, Cuthbertson AGS, et al (2016) Current SWD IPM tactics and their practical implementation in fruit crops across different regions around the world. J Pest Sci 89:643ï 651. doi: 10.1007/s10340-016-0737-8

Hebert PDN, Cywinska A, Ball SL, deWaard JR (2003) Biological identifications through DNA barcodes. Proc Biol Sci 270:313ї 21. doi: 10.1098/rspb.2002.2218

Kanzawa T (1939) Studies on Drosophila suzukii mats. J Plant Prot 23:29:622.

Kanzawa T (1934) Studies on a fruit fly, Drosophila sp. (in Japanese). Kontyu 8:302ï 303.

Kottek M, Grieser J, Beck C, et al (2006) World Map of the Koppen-Geiger climate classification updated. Meteorol Zeitschrift 15:259ï 263. doi: 10.1127/0941-2948/2006/0130

Kozeretska IA, Protsenko A V., Afanasieva ES, et al (2008) Mutation processes in natural populations of Drosophila melanogaster and Hirundo rustica from radiation-contaminated regions of Ukraine. Cytol Genet 42:267ï 271. doi: 10.3103/S0095452708040099

Köppen W (1931) Grundriss der Klimakunde. Walter de Gruyter, Berlin, Leipzig. 
Lachaise D, Silvain JF (2004) How two Afrotropical endemics made two cosmopolitan human commensals: The Drosophila melanogaster-D. simulans palaeogeographic riddle. In: Genetica. pp 17 ï 39

Lee JC, Bruck DJ, Curry H, et al (2011) The susceptibility of small fruits and cherries to the spotted-wing drosophila, Drosophila suzukii. Pest Manag Sci 67:1358ї 1367. doi: 10.1002/ps.2225

Markert JA, Champlin DM, Gutjahr-Gobell R, et al (2010) Population genetic diversity and fitness in multiple environments. BMC Evol Biol 10:205. doi: 10.1186/1471-2148-10-205

Mitsui H, Takahashi KH, Kimura MT (2006) Spatial distributions and clutch sizes of Drosophila species ovipositing on cherry fruits of different stages. Popul Ecol 48:233ï 237. doi: 10.1007/s10144-006-0260-5

Múrias Dos Santos A, Cabezas MP, Tavares AI, et al (2016) tcsBU: a tool to extend TCS network layout and visualization. Bioinformatics 32:627ї 8. doi: 10.1093/bioinformatics/btv636

Murphy KA, West JD, Kwok RS, Chiu JC (2016) Accelerating research on Spotted Wing Drosophila management using genomic technologies. J Pest Sci doi: 10.1007/s10340-016-0741-Z

Pascual M, Chapuis MP, Mestres F, et al (2007) Introduction history of Drosophila subobscura in the New World: A microsatellite-based survey using ABC methods. Mol Ecol 16:3069ï 3083. doi: 10.1111/j.1365294X.2007.03336.x

Pimentel D, Zuniga R, Morrison D (2005) Update on the environmental and economic costs associated with alieninvasive species in the United States. Ecol Econ 52:273ї 288. doi: 10.1016/j.ecolecon.2004.10.002

Radionov DB, Prosenko O V., Andrievsky AM, et al (2011) Stability of genetic parameters in Drosophila melanogaster populations from Odesa. Cytol Genet 45:187ї 190. doi: 10.3103/S0095452711030091

Ramasamy S, Ometto L, Crava CM, et al (2016) The evolution of olfactory gene families in Drosophila and the genomic basis of chemical-ecological adaptation in Drosophila suzukii. Genome Biol Evol. doi: 10.1093/gbe/evw160

Reed DH, Frankham R (2003) Correlation between fitness and genetic diversity. Conserv Biol 17:230ї 237. doi: 10.1046/j.1523-1739.2003.01236.x

Rota-Stabelli O, Blaxter M, Anfora G (2013) Drosophila suzukii. Curr Biol 23:R8ї R9. doi: 10.1016/j.cub.2012.11.021

Saguez J, Lasnier J, Vincent C (2013) First record of Drosophila suzukii in Quebec vineyards. J Int Sci Vigne Vin 47:69ї 72.

Serga S V., Maistrenko OM, Rozhok AI, et al (2015) Colonization of a temperate-zone region by the fruit fly Drosophila simulans (Diptera: Drosophilidae). Can J Zool 93:799ï 804. doi: 10.1139/cjz-2015-0018

Serga S, Maistrenko O, Rozhok A, et al (2014) Fecundity as one of possible factors contributing to the dominance of the wMel genotype of Wolbachia in natural populations of Drosophila melanogaster. Symbiosis 63:11ї 17. doi: 10.1007/s13199-014-0283-1

Tamura K, Stecher G, Peterson D, et al (2013) MEGA6: Molecular evolutionary genetics analysis version 6.0. Mol Biol Evol 30:2725ї 2729. doi: 10.1093/molbev/mst197

Tochen S, Dalton DT, Wiman N, et al (2014) Temperature-Related Development and Population Parameters for Drosophila suzukii (Diptera: Drosophilidae) on Cherry and Blueberry. Environ Entomol 43:501ї 510. doi: 10.1603/EN13200

Tochen S, Walton VM, Lee JC (2016a) Impact of floral feeding on adult Drosophila suzukii survival and nutrient status. J Pest Sci 89:793ї 802. doi: 10.1007/s10340-016-0762-7

Tochen S, Woltz JM, Dalton DT, et al (2016b) Humidity affects populations of Drosophila suzukii (Diptera: Drosophilidae) in blueberry. J Appl Entomol 140:47ï 57. doi: 10.1111/jen.12247

Tosevski I, Milenkovic S, Krstic O, et al (2014) Drosophila suzukii (Matsumura, 1931) (Siptera: Srosophilidae): A new invasive pest in Serbia. Zast bilja 65:99ï 104. doi: 10.5937/zasbilj1403099T

Walsh DB, Bolda MP, Goodhue RE, et al (2011) Drosophila suzukii (Diptera: Drosophilidae): Invasive Pest of Ripening Soft Fruit Expanding its Geographic Range and Damage Potential. J Integr Pest Manag 2:1ї 7. doi: 
Wang X-G, Stewart TJ, Biondi A, et al (2016) Population dynamics and ecology of Drosophila suzukii in Central California. J Pest Sci 89:701i 712. doi: 10.1007/s10340-016-0747-6

Wiman NG, Dalton DT, Anfora G, et al (2016) Drosophila suzukii population response to environment and management strategies. J Pest Sci doi: 10.1007/s10340-016-0757-4

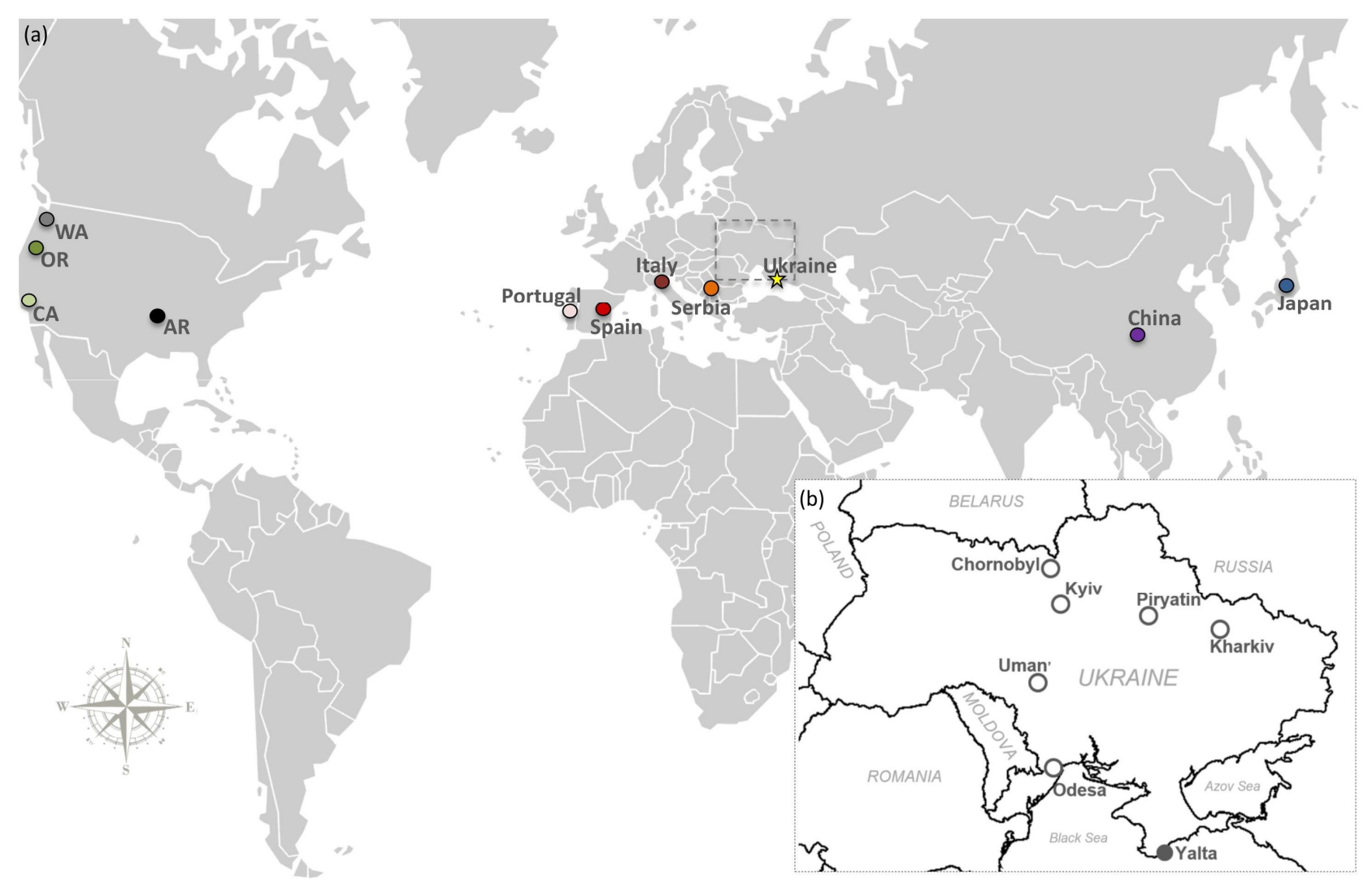

Fig. 1 Geographical distribution of D. suzukii sampling localities. (a) World map with sampling localities of COI analyzed specimens derived from GenBank (Online Resource HGB1), denoted with a circles. A star symbol represents location with specimens sequenced in the present study. Color of the symbols correspond to the sampling locations in the haplotype network (Fig.2). (b) Seven locations across Ukraine surveyed for Drosophila species. Sampling locations are denoted by open and closed circles, closed circle (Yalta, Crimea) indicates the location where specimens of $D$.

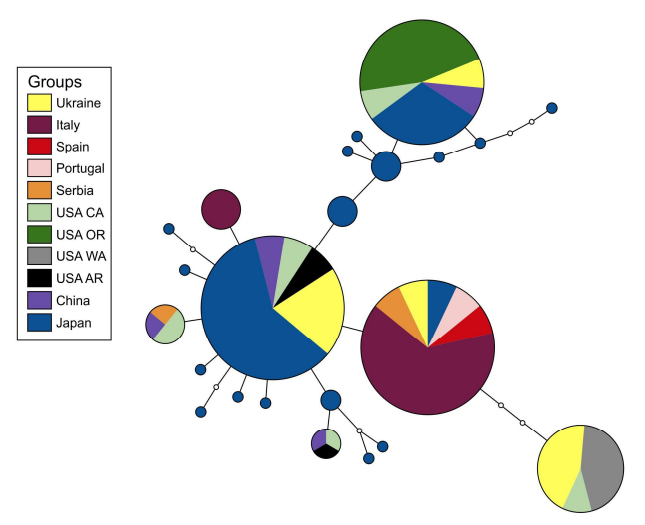

Fig. 2 Haplotype network of COI gene fragment (606 bp) for D. suzukii sampled from Ukraine, Europe, the USA, China and Japan (see GenBank accession numbers and number of samples in Online Resource HGB1). Colors indicate sample 
356 locations. Small white circles represent undetected intermediate haplotypes and each line corresponds to a mutational step. The area of the circles represent the amount of identical COI gene sequences used in the alignment

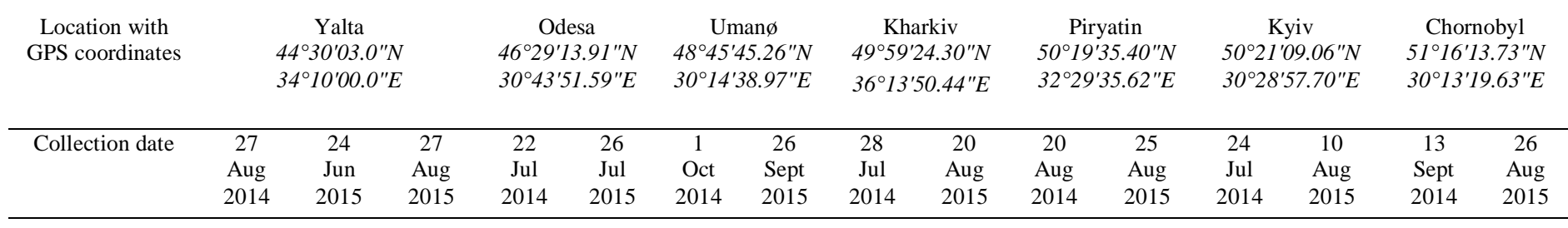

D. suzukii

11

$10 \quad 1$

358 Table 1 Sample locations with GPS coordinates, sampling dates, total number of D. suzukii individuals collected from

359 the Ukraine during 2014 and 2015 